\title{
Ultra-rare variants point to new autism candidate genes
}

\author{
BY NIKO MCCARTY
}

26 JULY 2021

\section{Listen to this story:}

https://www.spectrumnews.org/wp-content/uploads/2021/07/audiod603cef9-c7d3-43e5-a256-ac3752bfd037-encodings.mp3

An analysis of whole-genome sequences from thousands of families - one of the largest such studies to date — has linked 163 new genes to autism. The study appeared today in Nature Genetics.

The newly linked genes harbor ultra-rare inherited genetic variants - alterations to the genome that were passed from non-autistic parents to their autistic offspring. The variants are so rare that each one is found in only a single family.

Most previous genome sequencing studies for autism have focused on spontaneous, or de novo, mutations - genetic alterations that appear in a child but not in either parent. Those mutations are relatively easy to detect and have implicated hundreds of genes in autism.

"The unique part about this paper is that they're looking at a type of genetic variant that has not been studied so much in autism," says Sagiv Shifman, professor of genetics at The Hebrew University of Jerusalem, Israel, who was not involved in the study. "De novo mutations are just easier to study. They are low-hanging fruits."

\section{Deeper data:}

The new work draws on sequences from 4,364 autistic people and their non-autistic siblings and parents from five datasets, some of which are not yet published.

Although the datasets include whole genomes, the researchers focused their analysis initially on 


\section{Spectrum | Autism Research News}

https://www.spectrumnews.org

ultra-rare variants predicted to disrupt protein-coding genes.

"We thought we'd have a slightly better chance of detecting some signal in protein-coding genes over other types of variation that are not as well understood," says study investigator Amy Wilfert, senior research fellow in Evan Eichler's lab at the University of Washington in Seattle.

Autistic children were 1.2 to 1.3 times more likely than their non-autistic siblings to inherit an ultrarare variant in genes previously linked to autism, the researchers found. They replicated the finding in an additional set of whole-exome sequences from 6,453 autistic children and their families.

These changes account for about 4.5 percent of autism risk, the researchers estimate - a figure comparable to that for de novo mutations, suggesting that rare inherited variations are an understudied but important contributor to autism.

"In terms of population risk, these types of genetic variants can contribute almost as much as more well-known risk factors, such as de novo mutations," Wilfert says.

The findings also align with prior studies that looked at inherited variations but in data limited to the exome, or protein-coding regions of the genome, from a smaller number of families.

"This study gives me great confidence that the whole genome sequencing sets that we're generating are of high quality and worth analyzing. They get the same results from whole genome sequencing that others are getting from the exome alone," says David Cutler, associate professor of human genetics at Emory University School of Medicine in Atlanta, Georgia, who was not involved in the work. "We're likely walking down the correct path to understanding autism."

\section{Risk convergence:}

A second analysis homed in on genes with ultra-rare variants that appear to be intolerant to mutation and that have not been linked to autism in studies of de novo mutations. It identified 163 genes that when altered may increase a person's chances of having autism. None of these genes appear in SFARI Gene, a database that catalogues more than 1,000 genes with ties to the condition. (The database is funded by the Simon's Foundation, Spectrum's parent organization.)

Nearly half of the genes identified in the new study are involved in key cellular processes, such as protein regulation. Many are also expressed in specific types of cells in the human cortex, or outer layer of the brain, during development.

"It was really exciting to see that the gene candidates we identified are distinct from the genes that have been identified in de novo mutation studies to date," Wilfert says. "They do end up kind of converging on the same networks, which suggests shared pathways, but distinct genes are at play." 


\section{Spectrum | Autism Research News}

https://www.spectrumnews.org

Though the researchers looked solely at protein-coding genes, their datasets include the entire genome - including all the letters in between genes - for thousands of families. That data will be a useful starting point for other teams hoping to understand how regulatory, or noncoding, portions of the genome also contribute to autism, Cutler and Shifman say.

The full data will be uploaded to the database of Genotypes and Phenotypes, Wilfert says, so that other researchers can explore and ask new questions.

Cite this article: https://doi.org/10.53053/ECLI9432 